\title{
Gödel's Hermeneutics of the Relationship between Relativity Theory and Idealistic Philosophy
}

\author{
괴델이 해석하는 상대성이론과 관념론철학의 관계
}

\author{
Hyun Woosik 현우식
}

\begin{abstract}
This interdisciplinary study explores Gödel's hermeneutics of the relationship between relativity theory and idealistic philosophy in terms of time. For Gödel, Einstein's contribution to the physical realization of idealistic philosophy would be remarkable. We start with a historical background around Gödel's paper for Einstein(1949a). From the perspective of Gödel's cosmology, the second part addresses the relative nature of time, and the next then investigates the rotating model of universes. Gödel's own results show that the temporal conditions of relativity and idealistic philosophy are satisfiable in the mathematical model of rotating universes. Thus, it could be asserted to travel into any region of the past, present or future, and back again.
\end{abstract}

Keywords: Gödel, Einstein, relativity theory, idealistic philosophy, rotating universe; 괴델, 아인슈타인, 상대성이론, 관념론철학, 회전 우주.

MSC: A30, E20

\section{1 역사적 배경}

이 연구는 괴델[5]의 "A remark about the relationship between relativity theory and idealistic philosophy (1949a)" 를 중심으로 상대성이론, 관념론철학, 그리고 상호관계에 대한 괴델의 해석학을 고찰하고 그 의미를 재조명하기 위한 것이다. 이 연구에서 다루는 괴 델의 해석은 쉴프(Paul Schilpp, 1897-1993)가 편집한 책『아인슈타인: 철학자-과학자』에 발표된 소논문을 중심으로 이루어 질 것이다[4]. ${ }^{1)}$ 이 책은 특별히 아인슈타인(1879-1955) 의 70회 생일 (1949년 3월 14일)을 축하하기 위해 구상된 것이었다. ${ }^{2)}$ 쉴프 교수는 1946년에 괴델에게 아인슈타인을 위한 원고를 처음 요청했다 $[2,14]$. 이에 대하여 괴델이 다섯 번의 수정

Hyun Woosik: Hoseo Univ. E-mail: godel@hoseo.edu

Receiveded on Dec. 5. 2013, revised on Feb. 4. 2014, accepted on Feb. 10. 2014.

1) Albert Einstein, Philosopher-Scientist, Library of Living Philosophers, vol. 7, Evanston: Library of Living Philosophers, 1949, 557-562.

2) 당시 쉴프 교수는 '살아있는 철학자 시리즈' 의 편집자였고 노스웨스턴 대학교의 철학교수였다. 
끝에 출판에 동의한 글이『아인슈타인 : 철학자-과학자』을 통하여 발표된 것이었다.

괴델은 아인슈타인 외에도 러셀 (B. Russell), 카르납(R. Carnap), 포퍼 (K. Popper)에 관한 기고를 요청 받았었다. 이 중에서 괴델의 글이 공식적으로 출판된 것은 아인슈타인의 경우가 유일하다. 괴델은 철저하게 준비하여 글을 썼다. 그리고 그는 발표를 최종 결정하기 전에 여러 가지 면에서 숙고를 거듭했던 신중한 인물이었다. 예를 들면, 괴델은 카르납의 입장에 대한 비판적 논문 ‘수학은 언어의 구문론인가?' 를 작성했고 적어도 여섯 개 이상의 원고를 작성했다. 그러나 괴델은 결국 논문 발표를 하지 않았다. 괴델이 논문에 대하여 만족 하지 않는다는 표면적 이유가 있었으나, 동시에 카르납을 배려한 내면적 이유도 있었다. 그런 점에서 아인슈타인에 대한 괴델의 글이 발표된 사건 자체가 이 글이 가지는 역사적 중요성과 가치를 잘 보여준다.

괴델에 의하면, 괴델이 처음 아인슈타인을 만났던 것은 1933년이다[2]. 이 때 괴델과 아 인슈타인을 서로 소개해 준 사람은 오펜하임 (Paul Oppenheim) 이었다. ${ }^{3)}$ 괴델은 1942년 부터 아인슈타인과 가까운 사이가 되었다. 두 사람이 서로에게 큰 호감을 가지고 있던 것은 분명하다. 그래서 두 사람의 대화는 아인슈타인이 사망하는 1955 년까지 지속되었다. 괴델과 아인슈타인의 이야기는 수학, 물리학, 신학, 철학, 정치 등을 포함한 폭넓은 주제를 자유롭게 다룬 대화였다[2, 14, 15].

괴델과 마찬가지로 아인슈타인도 괴델과의 대화를 즐겼다. 이에 대하여 괴델 자신의 해석에 의하면, (1) 괴델이 빈번하게 아인슈타인의 의견과 반대되는 의견을 가지고 있었다는 점과 (2) 괴델이 아인슈타인에게 동의하지 않는 것을 숨기려고 하지 않았다는 점이 그 이유에 포함되 었다[2, p. 176]. 서로의 성격이나 스타일 등 여러 가지로 다르고 대조되는 면모가 많았으나, 괴델과 아인슈타인은 지적인 대화를 퍽 즐겼고, 서로를 더욱 깊이 이해하는 친밀한 사이가 되었다. 예를 들면, 1848 년 7월 12일자 서신에서 괴델은 어머니 마리안네 (Marianne) 에게 거의 매일 아인슈타인을 만나고 있다고 전했다[14, p. 32]. 또한 1955년 4월 18일자 서신에서 괴델은 아인슈타인의 죽음 이후 큰 충격을 받고 있음을 어머니에게 고백하고 있다[14, p. 33].

두 사람이 서로 인정하고 존경한 근거는 무엇이었을까? 아인슈타인은 수학 분야에도 자신과 같은 사람이 있다는 것을 발견했고, 괴델은 물리학 분야에도 자신과 같은 사람이 있다는 것을 발견했기 때문이다. 두 거장은 물리학과 수학의 부분적 문제 보다는 오히려 그 기초가 되는 문제가 무엇이며 어떤 의미가 있는 지에 더 깊은 매력을 느끼고 있었다는 점에서 동감했을 것이다[16].

괴델의 친구 모르겐슈테른(Oskar Morgenstern) 교수는 오스트리아 외무장관 크라이스키 (B. Kreisky)에게 보내는 서신 (1965년 10월 25일자)에서 괴델과 아인슈타인의 친밀한 관계 를 다음과 같이 알려주고 있다[14, p. 31].

3) 독일 출신의 오펜하임은 독일을 떠나 프린스턴에 정착하여 살던 화학자이자 철학자였다. 
"아인슈타인이 나에게 자주 말한 바에 의하면, 아인슈타인은 괴델과 토의하기 위해서 계속 괴델과 친구로 지내고 싶어 했다고 합니다. 한번은 아인슈타인이 자신의 연구는 이제 더 이상 큰 의미가 없으며, 고등학문연구소를 가는 이유는 단지 "괴델과 함께 집으로 걸어올 수 있는 특혜를 누리기 위해서라고(um das Privileg zu haben, mit Gödel zu Fuss nach Hause gehen zu dürfen)” 내게 말했습니다.”

\section{2 시간의 본성}

괴델은 ‘시간의 본성' 에 관하여 묻는다. 괴델에 의하면 시간은 신비로운 대상이며 자기모순적 인 대상이다. 다른 한편, 시간은 우주의 기초를 형성하는 대상이다. 그러므로 우리의 존재를 형성하는 기초이다. 여기에서 아인슈타인의 상대성 이론은 우리가 시간을 이해하는 일에 큰 공헌을 한다는 것이다[3]. 특수상대성이론의 출발점은 바로 시간의 새롭고 놀라운 속성을 발견하는 것으로 전개된다. '동시성의 상대성' 이 그 좋은 예이다. 여기에서 사건 $\mathrm{A}$ 와 사건 $\mathrm{B}$ 가 동시적이라고 하는 주장은 객관적 의미를 잃게 된다. 왜냐하면, 관측자가 달라지면 그 동시성은 유지될 수 없기 때문이다. 즉, 같은 논리에 의해서 다른 관측자에 의하면 사건 $\mathrm{A}$ 와 사건 $\mathrm{B}$ 는 동시적이 아니라고 주장될 수 있다. 동시성은 절대적이라고 주장될 수 없다.

동시성의 상대성은 시간의 본성에 대한 결론이 쉽게 내려질 수 있는 것이 아님을 잘 보여 준다. 시간의 본성에 대한 철학자들의 견해는 다양했다. 관념론철학에 의하면 변화의 객관 성은 부정된다. 이 때, 변화는 우리가 가진 특별한 감각에 의한 환상이나 현상으로 간주된다. 괴델은 특별히 현대 관념론자들을 주목한다. 엄밀히 말하자면, 괴델이 말하는 관념론철학의 범위는 칸트의 관념론에 집중되는 것으로 보는 것이 타당하다[13]. 괴델은 칸트의『순수이성 비판』(Critique of pure reason 1787, A37, B54)을 인용한다[4, p. 558]. 여기에서 시간은 감각의 한 형식이다. 감각 내에서는 시간이 생기지 않는다는 것이다. 괴델은 이러한 논리가 상대성이론에서 말하는 상황과 잘 어울린다고 보았다. 예를 들면, 물체의 우주선(the world line)에 대한 경사각은 민코스키 공간(Minkowski space) 내에서 상대적 경사각으로 해석될 수 있다.

괴델은 다음과 같이 논증을 시작한다. 변화는 가능하다. 단 시간의 경과를 통해서만 가능하 다. 즉 변화의 조건은 '시간의 경과' (the lapse of time) 이다. 그러나 객관적인 시간의 경과가 존재한다는 것은 실재 (reality) 가 무한성으로 구성된다는 것을 의미한다. 여기에서 말하는 무한성은 바로 “현재” 라는 층 (layers) 의 무한성이다. 그러므로 현재는 연속적으로 존재하게 된다.

괴델은 객관적으로 시간이 경과한다는 것이 무의미하다는 주장에 의해서 변화의 관념에 대한 관념론적 입장을 취할 수 있다고 본다[4, p. 558]. 오직 현재만이 존재하므로, (1) 객관적으로 
시간이 경과한다는 관념을 논하는 것이 무의미하다는 주장도 할 수 있다. 현재만이 존재한다면, (2) 변화의 관념은 자기 모순적 관념이라고 주장할 수도 있다. 객관적 시간의 경과의 가능성을 부정하면, 실재 존재하는 것을 부정하는 것이 된다. 그렇다면 객관적 시간의 경과의 관념이 의미 없는 관념이거나 자기 모순적인 관념으로 고려되는 것과 큰 차이가 없다.

상대성이론에 의해 동시성이 상대적인 동시성이라면 실재는 객관적으로 결정되는 방법에 의해서 층들 속으로 분리될 수 없다[4, p. 559]. 각각의 관측자는 고유한 “현재” 의 집합을 가지기 때문이다. 즉, 층의 다양한 시스템을 전제한다고 해도 객관적 시간의 경과를 표상할 수 없다. 관념론철학자들은 시간의 경과란 단지 생각에 의해 구성된 것이라 주장한다. 관념 론철학자들은 결코 객관적인 시간의 경과를 받아들일 수 없다. 괴델에 의하면 시간의 경과는 확정적 방법에 따른 경과가 아니다. 시간의 경과는 확정된 색을 가지고 있지 못한 대상이 색을 가지고 있다고 말하는 것처럼 불합리한 것을 의미한다.

다른 속도로 운동하는 모든 관측자들이 완전하게 등가라고 할 때, 그 등가성 (equivalence) 은 특수상대성이론의 추상적인 시공간 틀 내에서 존재한다. 그리고 그 등가성은 일반상대성이 론의 어떤 진공 세계 내에서 존재한다. 이 주장이 부정될 수 있는가? 괴델은 부정될 수 없다고 생각한다. 왜냐하면 물질의 존재는 대부분 상이한 관측자들의 등가성을 파괴한다. 시공간의 특정한 곡률도 상이한 관측자들의 등가성을 파괴한다. 동시에 일부 관측자와 다른 관측자들을 구별한다. 그러므로 위의 주장은 부정될 수 없다는 것이다.

괴델은 절대적 시간이 객관적으로 경과한다는 주장이 정당화될 수 없음을 논증한다[4, 559-560]. 모든 관측자들의 국소적 시간(the local time)이 하나의 세계에서 서로 일치한다고 할 때, 이 시간은 하나의 '진정한' 시간이고 객관적으로 경과하는 시간이라고 생각할 수 있다. 반면, 다른 관측자들의 측정 결과가 일치하지 않는다면 객관적으로 경과하는 시간이라고 생각할 수 없다. 이 경우 운동은 물질 운동의 평균상태와 상대적이다. 여기에서 상대적 운동은 측정의 과정에 근거한다.

\section{3 회전하는 우주}

괴델에 의하면 절대 시간을 정의하는 작업은 필요하지 않다. 특정한 측정자의 국소적 시간은 하나의 세계 시간 내에서 서로 조정될 수 없기 때문이다. 변화의 비객관성 (non-objectivity) 에 관한 괴델의 주장은 우리가 사는 우주에 적용될 수 있다. 괴델에 의하면, 이 우주 내에서 시간의 조건은 다른 놀라운 특성들을 보여준다. 그리고 이 특성들이 관념론적 입장을 더 강화해 줄 수 있다고 괴델은 해석한다.

여기에서 괴델이 말하는 시간적 조건이란 무엇인가? 괴델은 우리의 우주 내에서 '관성의 컴퍼스(the compass of inertia)' 가 은하계 전체에 대하여 상대적으로 회전한다고 해석한다. 이러한 조건을 만족하는 우주를 “회전하는 우주(rotating universes)” 라고 부른다. 즉 괴델은 
회전하는 우주를 전제한다. 괴델이 말하는 우주는 다음 세 가지를 만족해야 하는데, (1) 정적 (static) 상태를 추가적 속성으로 가지고 있고, (2) 공간적으로 동질적 (homogeneous)이며, (3) 우주 상수가 0보다 작다는 것이 그 조건이다[4, p. 560, 각주10]. 괴델은 이러한 내용을 이미 수학적으로 증명하여 발표했다[6]. ${ }^{4}$. 괴델은 물질의 세계선(the world line) 위에서 직교성 (orthogonality) 을 가지는 삼차원 공간에 대한 단일한 매개변수 시스템은 존재하지 않는다고 본다. 그리고 이러한 시스템이 존재하지 않는다는 결론은 곧 ‘관성의 컴퍼스' (compass of inertia)에 대하여 상대적인 물질이 회전한다는 결론과 논리적으로 같다는 주장이 괴델에 의해 증명된다.

1949년 괴델이 발표한 새로운 상대론적 우주론에 대한 공헌은 그의 독창적 해법에 있었 다[6]. 아인슈타인의 일반상대성이론 방정식과 먼 은하들의 적색 편이에 대한 관측 결과는 서로 모순되지 않는 것으로 해석되었다. 이것을 위한 모델은 팽창 모델인데, 회전하지 않는 팽창 우주 모델이었다. 즉 공간적으로는 동질적 (homogeneous) 이고 등방적 (isotropic) 인 모델이었다. 그러나 괴델은 최초로 회전하는 우주의 모델을 제시한다. 괴델의 회전하는 우주 모델은 마치 충분하게 넓은 곡선 내에서 우주비행선이 원운동을 하는 것과 같이 이해될 수 있다. 그러므로 이 회전하는 우주 내에서는 과거의 영역, 현재의 영역, 미래의 영역으로 여행 하는 것이 가능하다. 그리고 다시 돌아오는 여행도 가능하다. 이러한 상태는 합리적이지 못한 것으로 여겨질 수 있으나, 괴델은 실재 우주의 구조가 이러한 구조라고 주장한다[4, 560-561].

괴델의 주장은 아인슈타인의 일반상대성 이론이 함의하고 있는 새롭고 중요한 의미를 밝혀주었다. 마흐의 법칙 (Mach's principle) 에 의하면, 절대 공간을 부정하면 우주 내에서 물질은 다른 물질과 상대적인 관성을 가져야한다. 그렇다면 국소적 관성틀(the local inertia frame) 은 회전할 수 없다. 그러나 괴델은 아인슈타인의 상대성이론의 장 방정식에 의해서 이 국소적 관성틀에 대하여 회전하는 은하를 가질 수 있다는 해법을 제공한 것이다. 이 결과에 의하면, 아인슈타인의 상대성이론은 마흐의 법칙으로부터 자유롭게 될 수 있다[11]. 이러한 공헌을 인정받아, 괴델은 1951 년 3월 14 일 제 1 회 아인슈타인 상의 수상자가 되었다. 괴델의 회전하는 우주론은 다양한 논쟁을 제공하고 있으며, 이론물리학과 우주과학 분야에서 다양한 연구가 현재까지 진행되고 있다[11, 17, 1, 12].

괴델은 상대성이론이 함의하는 놀라운 시간의 의미를 세상에 알리고자 했다. 그래서 그는 거의 8년 만에 대중 앞에서 '일반상대성에서 회전하는 우주("Rotating universes in general relativity”') 라는 제목의 강연을 행하게 된다[7]. 1950년 8월 31일 세계수학자대회 (the International Congress of Mathematicians, Cambridge, Massachusetts)에서 초청강연 자 괴델은 상대성이론과 관념론철학의 관점에서 ‘시간의 본성’ 을 수학적으로 풀이하고 해석

4) An Example of a New Type of Cosmological Solutions of Einstein's Field Equations of Gravitation, Review of Modern Physics 21 (1949), 447-450 
했다.

\section{4 결어}

괴델의 논문 (1949a)은 아인슈타인에 대한 특별한 우정을 보여주는 것 외에도, 시간에 대한 괴델의 해석, 괴델의 회전하는 우주론, 그리고 물리학과 철학이 공유할 수 있는 관계에 대한 괴델의 해석을 보여준다. 괴델 논문의 결론에는 다음과 같은 명제가 포함된다[4, p. 561].

"우주의 시간에 대한 가능한 모든 정의에 대하여, 우주의 과거로 여행할 수 있다.

여기에서 과거는 해당 정의에 따른 과거이다.”

이러한 결과는 객관적으로 시간이 경과한다는 전제를 가지면 이 우주 내에서 정당화되는 모든 것을 잃을 수 있다는 것을 보여준다. 왜냐하면 시간이 경과한다는 전제를 가지면 항상 관측자가 존재할 수 있어야 하는데, 시간의 경과를 경험한 관측자에 상응하는 객관적 경과는 없기 때문이다. 예를 들면, 모든 관측자가 객관적으로 동시에 존재해야 하는 조건을 만족하는 관측자들이 존재할 수 없다는 것이다.

괴델의 결론은 다음과 같다[4, p. 562]. 첫째, 팽창 회전하는 해법 (expanding rotating solutions)에 의해서 우리의 우주는 설명될 수 있다.5) 이러한 우주에서 절대적 시간은 존재할 수 없다. 괴델은 우리의 우주가 팽창하면서 회전하는 우주라는 사실이 가능하다고 믿었다. 둘째, 구별될 수 있는 절대 시간은 없다. 그러므로 객관적 시간의 경과는 존재하지 않는다. 이는 우주의 자연 법칙과 양립가능하다. 이 점에서 시간에 대한 괴델의 해석은 그가 해석하는 칸트의 관념론철학과 연속성을 지닌다 $[9,10]$.

괴델의 해석에 의하면, 아인슈타인의 상대성이론의 시간 해석은 관념론의 철학적 해석과 논리적으로 양립가능하다. 여기에서 객관적 시간의 존재는 증명될 수 없다. 따라서 객관적 시간의 경과와 객관적 변화의 존재도 증명될 수 없다. 시간은 상대적 시간이다. 괴델에게 상대적 시간의 조건은 회전하는 우주 모델 안에서 수학적으로 만족될 수 있는 것이다. 이 회전하는 우주 내에서는 시간 여행이 가능하다.

\section{참고 문헌}

1. J. Barrow, Gödel and Physics in Kurt Gödel and the Foundations of Mathematics: Horizon of Truth, M. BaAz, C. Papadimitriou, H. Putnam, D. Scott, C. Harper, Jr. (eds.), Cambridge: Cambridge University Press, 2011, 255-276.

2. J. Dawson, Logical Dilemma: The Life and Work of Kurt Gödel, Massachusetts: A K Peters, 1997.

5) 팽창을 배제한 회전하는 해법은 정적인 속성을 가지고 있으므로 멀리 있는 대상에 대한 적색 편이 (red-shift) 를 설명하지 못한다. 
3. R. Feynman, 16-1 Relativity and Philosophers in The Feynman Lectures on Physics I, R. Feynman, R. Leighton, M. Sands, New York: Addison-Wesley Publishing Company, 1963.

4. K. GöDEL, A remark about the relationship between relativity theory and idealistic philosophy in Albert Einstein, Philosopher-Scientist, Paul Schilpp (ed.), La Salle: Open Court, $1949,557-562$.

5. K. GöDEL, A remark about the relationship between relativity theory and idealistic philosophy (1949a) in Kurt Gödel Collected Works II: Publications 1938-1974, S. Feferman, J. Dawson, Jr., S. Kleene, G. Moore, R. Solovay, J. van Heijenoort (eds.), New York: Oxford University Press, 1990, 202-207.

6. K. GöDEL, An example of a new type of cosmological solutions of Einstein's field equations of gravitation (1949) in Kurt Gödel Collected Works II: Publications 1938-1974, S. Feferman, J. Dawson, Jr., S. Kleene, G. Moore, R. Solovay, J. van Heijenoort (eds.), New York: Oxford University Press, 1990, 190-198.

7. K. GöDEL, Rotating universes in general relativity theory (1952) in Kurt Gödel Collected Works II: Publications 1938-1974, S. Feferman, J. Dawson, Jr., S. Kleene, G. Moore, R. Solovay, J. van Heijenoort (eds.), New York: Oxford University Press, 1990, 208-216.

8. K. GöDEL, Lecture on rotating universes $\left({ }^{*} 1949 b\right)$, Kurt Gödel Collected Works III: Unpublished Essays and Lectures, S. Feferman, W. Goldfarb, C. Parsons, R. Solovay (eds.), New York: Oxford University Press, 1995, 269-287.

9. K. GöDEL, Some observations about the relationship between theory of relativity and Kantian philosophy $\left({ }^{*} 1946 / 9-B 2\right)$ in Kurt Gödel Collected Works III: Unpublished Essays and Lectures, S. Feferman, W. Goldfarb, C. Parsons, R. Solovay (eds.), New York: Oxford University Press, 1995, 230-246.

10. K. GöDEL, Some observations about the relationship between theory of relativity and Kantian philosophy $\left({ }^{*} 1946 / 9-C 1\right)$ in Kurt Gödel Collected Works III: Unpublished Essays and Lectures, S. Feferman, W. Goldfarb, C. Parsons, R. Solovay (eds.), New York: Oxford University Press, 1995, 247-259.

11. S. Hawking, Introductory note to 1949 and 1952 in Kurt Gödel Collected Works II: Publications 1938-1974, S. Feferman, J. Dawson, Jr., S. Kleene, G. Moore, R. Solovay, J. van Heijenoort (eds.), New York: Oxford University Press, 1990, 189-190.

12. W. Rindler, Gödel, Einstein, Mach, Gamow, and Lanczos: Gödel's Remarkable Excursion into Cosmology in Kurt Gödel and the Foundations of Mathematics: Horizon of Truth, M. Baaz, C. Papadimitriou, H. Putnam, D. Scott, C. Harper, Jr. (eds.), Cambridge: Cambridge University Press, 2011, 185-211.

13. H. Stein, Introductory note to 1949a in Kurt Gödel Collected Works II: Publications 19381974, S. Feferman, J. Dawson, Jr., S. Kleene, G. Moore, R. Solovay, J. van Heijenoort (eds.), New York: Oxford University Press, 1990, 199-201.

14. H. WANG, Reflections on Kurt Gödel, Cambridge: The MIT Press, 1987.

15. H. WANG, A Logical Journey: From Gödel to Philosophy, Cambridge: The MIT Press, 1996.

16. Hyun Woosik, Gödel on the Foundations of Mathematics, The Korean Journal for History of Mathematics 20(3) (2007), 17-26. 
17. P. Yourgrau, Gödel Meets Einstein: Time Travel in the Gödel Universe, La Salle: Open Court, 1999. 